\title{
Trajectories of bright stars at the Galactic Center as a tool to evaluate a graviton mass
}

\author{
Alexander Zakharov ${ }^{1,2,3,4, \star}$, Predrag Jovanovićc ${ }^{5, \star}$, Dusko Borka ${ }^{6, \star \star \star}$, and Vesna Borka \\ Jovanović6, \\ ${ }^{1}$ Institute of Theoretical and Experimental Physics, 117259 Moscow, Russia \\ ${ }^{2}$ Bogoliubov Laboratory for Theoretical Physics, JINR, 141980 Dubna, Russia \\ ${ }^{3}$ National Research Nuclear University MEPhI (Moscow Engineering Physics Institute), 115409, Moscow, \\ Russia \\ ${ }^{4}$ North Carolina Central University, Durham, NC 27707, USA \\ ${ }^{5}$ Astronomical Observatory, Volgina 7, 11060 Belgrade, Serbia \\ ${ }^{6}$ Atomic Physics Laboratory (040), Vinča Institute of Nuclear Sciences, University of Belgrade, P.O. Box 522, \\ 11001 Belgrade, Serbia
}

\begin{abstract}
Scientists worked in Saint-Petersburg (Petrograd, Leningrad) played the extremely important role in creation of scientific school and development of general relativity in Russia. Very recently LIGO collaboration discovered gravitational waves [1] predicted 100 years ago by A. Einstein. In the papers reporting about this discovery, the joint LIGO \& VIRGO team presented an upper limit on graviton mass such as $m_{g}<1.2 \times 10^{-22} \mathrm{eV}[1,2]$. The authors concluded that their observational data do not show violations of classical general relativity because the graviton mass limit is very small. We show that an analysis of bright star trajectories could bound graviton mass with a comparable accuracy with accuracies reached with gravitational wave interferometers and expected with forthcoming pulsar timing observations for gravitational wave detection. This analysis gives an opportunity to treat observations of bright stars near the Galactic Center as a tool for an evaluation specific parameters of the black hole and also to obtain constraints on the fundamental gravity law such as a modifications of Newton gravity law in a weak field approximation. In that way, based on a potential reconstruction at the Galactic Center we give a bounds on a graviton mass.
\end{abstract}

\section{Brief history of GR development in Russia}

It is well-known that general relativity (GR) was discovered in November 1915 [3, 4] (see also [5-9] for a more detailed historical description of the issue). The gravitational waves in the framework of GR have been discussed in A. Einstein paper [10] published on June 22, 1916. The Quarks-2016 was organized in Pushkin near Saint-Petersburg. Four Saint-Petersburg institutions organized the seminar, namely, Petersburg Nuclear Physics Institute of NRC "Kurchatov Institute", Saint-Petersburg

\footnotetext{
$\star$ e-mail: zakharov@itep.ru

$\star \star$ e-mail: pjovanovic@aob.rs

$\star \star \star$ e-mail: dusborka@vin.bg.ac.rs

$\star \star \star \star$ e-mail: vborka@vinca.rs
} 
State University, Peter the Great Saint-Petersburg Polytechnic University and Saint-Petersburg Scientific Center of RAS. Saint-Petersburg is practically the birthplace of Soviet (Russian) GR school and perhaps the school started to work almost immediately after the revolution and World War I, when Vsevolod Frederiks returned to Russia in 1918 (in the same year the main Soviet review journal Uspekhi Fizicheskikh Nauk has been established). ${ }^{1}$. In 1918 Soviet Russia was in ruins after revolutions and World War I, financial and human losses were gigantic, but in spite of that a scientific life was going on. In 1921 Frederiks published three papers Physics - Uspekhi journal including a paper [22], where he described basic concepts of GR. Frederiks and Friedmann planned to write a book "Foundations of General Relativity", but unfortunately Friedmann died after severe typhoid in 1925 when he was only 37 and only the first chapter "Tensor calculus" has been written and published [23]. As V.A. Fock wrote [24]: "Alexander Alexandrovich Friedmann and Vsevolod Konstantinovich Frederiks were professors of Petrograd University (now Leningrad University) and they were the first scientists who started to teach Russian physicists (working in Petrograd) GR...Main speakers were V.K. Frederiks and A.A. Friedmann. Styles of their talks were rather different. Frederiks clearly understood physical aspects of theory ${ }^{2}$ and disliked to show mathematical calculations, while Friedmann placed emphasis on mathematics but not on physics. He aimed at mathematical rigor and paid attention to complete and precise form ulations of initial assumptions. Discussions between Frederiks and Friedmann were very interesting." Now it is clear that it was not strange that one of the most important solutions of Einstein equations has been found by A. A. Friedmann [25, 26] and now we live in the Friedmann universe. The hot Universe model (the Big Bang model) has been introduced by G. Gamov [27] who was A. Friedmann's student [28]. Conferences on general relativity and gravitation

\footnotetext{
${ }^{1}$ The crucial role of Frederiks in development of Russian science has been pointed out in the literature, for instance, V. P. Vizgin and V. Ya. Frenkel named their article as "Vsevolod Konstantinovich Frederiks as a pioneer of relativity and liquid crystal physics in USSR" [11]. After finishing high school Vsevolod Kostantinovich Frederiks continued his education in Europe as it was rather usual for young people from noble families if they decided to work in science or education [12, 13]. He selected the physical department of the Geneva University. There were two reasons to select namely the Geneva University, first, the Geneva location in a center of Western Europe is very convenient to visit other European scientific centers, second, the Geneva University had a very strong school of experimental solid state physics and electromagnetism and a famous scientist Charles-Eugene Guye was a professor and Director of Physical Institute at the Geneva University. Guye was Frederiks's supervisor. Earlier, Guye was a Privatdozent at Zurich Polytechnic where he was a teacher of A. Einstein. Starting in 1907 Guye and his assistants conducted wonderful experiments where they showed that electron masses depend on their speed [14-16] and experimentally confirmed predictions of special relativity. After defence of PhD thesis in Geneva Frederiks went to Göttingen to work since 1911 as an assistant of Woldemar Voigt who was Director of Physical Institute of the Göttingen University (Woldemar Voigt had a number of scientific titles, in particular, in 1913 he was elected a Honored Member of Imperial Saint-Petersburg University). Voigt was the first scientist who assumed universal speed of light in 1887 and tried to find transformations which keep an invariance of the wave equations [17] (see also [18, 19] for a more detailed discussion of the issue, in particular, differences between Voigt and Lorentz transformations). In summer 1914 W. Voigt left the Physical Institute Director position. In August 1914 World War I started and it was unexpected for many Russians staying in Europe at the time, after that Russian people (including V. Frederiks) had a status of civilian prisoners-of-war. It means that they have number of restrictions, in particular, Russian people have no right to have a job with wages from governmental institutions. In these circumstances, Frederiks has been resigned from the Physical Institute. However, Voigt asked authorities to permit Frederiks to work in laboratory. As a result of their joint studies in 1915 Voigt and Frederiks published an important paper on piezoelectricity [20], where the authors proved that Voigt model of piezoelectricity is more correct than a model proposed by W. C. Röntgen [21]. Since 1912 Hilbert was interested in mathematical aspects of theoretical physics and he had assistants as consultants on actual problems of physics. Duties of Hilbert's assistant were to report every day about the most important results in branches of physics which were interesting for Hilbert. Since it was impossible to obtain an official position and official wages from the Göttingen University for Frederiks as a citizen of enemy country, Hilbert offered Frederiks to be an assistant on physics and he paid an assistant salary from his own personal funds. The Hilbert's action had an important impact on development of general relativity, geometry and mathematical aspects of physics in Russia.

2 "When I asked question: "How do you formulate a law of motion of many bodies in GR?" he immediately answered: "Motion of bodies is determined by motion of singular points of metric tensor". It was said many years before derivations of equations of motion for system of bodies (as it was done in Einstein et al. and my papers in 1938 - 1939)" [24].
} 
play very important role in a development of the branch of science. One of the first conferenc ${ }^{3}$ was organized under supervision of W. Pauli in 1955 and it was a celebration of forty years since a creation of GR and fifty years since the discovery of special relativity. Two participants A.D. Alexandrov and V.A. Fock represented USSR where Alexandrov presented an invited plenary talk on mathematical aspects of GR. In 1955 A.D. Alexandrov was a rector of Leningrad University and corresponding member of Academy of Sciences of USSR. He was only 42 but he delivered a talk not because he had a high administrative position but because of his high scientific reputation. Unfortunately, now it is not common practice when young leading scientists are rectors of top universities. Academician A.D. Alexandrov had many outstanding students including famous G. Perelman who was last Alexandrov's student and a recipient of a number of the many prizes (including Fields medal and the Millennium prize) but he rejected to accept these prizes.

\section{Introduction}

Very recently long-term efforts of theorists and experimentalists have been led to a remarkable discovery of gravitational waves [1]. In June 2016 LIGO collaboration reported about the detection of the second gravitational wave event and one suspected case [29] (LIGO people reported about the result one week before the century jubilee since a publication of the first Einstein paper on gravitational waves [10]). ${ }^{4}$ This discovery represents a brilliant confirmation of general relativity and gives also a confirmation of black hole existence in binary systems. Different alternative theories of gravity, including massive graviton theories introduced by Fierz and Pauli [30] are a subject of intensive studies. Gravitational wave observations could constrain a graviton mass since in the case of massive graviton a gravitational wave signal is different from a signal of general relativity [31,32]. Analyzing and comparing observations with massive and massless models one could obtain a graviton mass constraint. Additionally, in the case if source of gravitational radiation signal is known one could detect a time delay of gravitational wave signal in respect to electromagnetic and neutrino signals. A slow progress in understanding of dark matter (DM) and dark energy (DE) problems stimulates a growth of interest to alternative theories of gravity. Nowadays, there are many alternative theories of gravity which have been proposed. In spite of the evident success of the conventional general relativity (GR) since after 100 years of its development there are necessity to cure its shortcomings. Also, perhaps there are deviations from Newtonian gravity at the Solar system scales $[33,34]$ in spite of a model of the thermal origin of the anomaly [35]. Some other alternative theories of Lorentz invariant massive gravity has been introduced [30], but scieniest found a number of problems with such theories such as existence of ghosts, vDVZ discontinuity [36-39]. Some of these problems can be overcame [39-48]. We have to note that some of alternative theories do not have the Newtonian limit in a weak gravitational field approximation. For example, Yukawa-like corrections have been obtained, as a general feature, in the framework of $f(R)$ theories of gravity [49]. A number of gravity theories have a Yukawa limit for a weak gravitational field. In this investigation, we will discuss observational consequences of massive gravity where one can expect a Yukawa type of weak gravitational field limit instead of Newtonian one. Experimental and observational ways to bound a graviton mass can be very different. Will considered an opportunity to evaluate a graviton mass analyzing a time delay in electromagnetic waves such as supernova or gamma-ray burst [32], or from from gravitational wave signal alone [31]. Some

\footnotetext{
${ }^{3}$ Now it is called GR0 conference according to the standard scientific chronology while the first GR conference (GR1) has been organized by Bryce De Witt in Chapel Hill (NC) in 1957.

${ }^{4}$ As one could see the LIGO collaboration reported about the discoveries of gravitational wave events in the time interval between one century jubilee since the GR discovery in November 1915 and one century since the first Einstein paper on gravitational waves published in June 1916.
} 
other suggestions are given in following references [50-55]. It was shown that speed of gravity practically coincides with speed of light [56-60], and it can be used to get constraints of a graviton mass. Constraints on the range $\lambda$ of Yukawa type force in Solar system has been considered [61] and one can derive the bound $\lambda>2.8 \times 10^{12} \mathrm{~km}$ [32] from these constraints assuming a natural modification of the Newtonian potential [32, 41]:

$$
V(r)=-\frac{G M}{(1+\delta) r}\left[1+\delta e^{-\left(\frac{r}{\lambda}\right)}\right],
$$

where $\delta$ is a universal constant. In our previous investigation [62] we found constraints on parameters of Yukawa gravity.

One more way that can be used to evaluate a graviton mass [63] is pulsar timing. An idea to use pulsar timing for gravitational wave detection has been proposed many years ago [64]. An analysis of the cross-correlation function between pulsar timing residuals of pulsar pairs could give an opportunity to detect gravitational waves $[65,66]$. With $90 \%$ probability, massless gravitons can be distinguished from gravitons heavier than $3 \times 10^{-22} \mathrm{eV}$ (Compton wavelength $\lambda_{g}=4.1 \times 10^{12} \mathrm{~km}$ ) [63]. Predictions show that if bi-weekly observation of 60 pulsars is performed for 5 years with a pulsar rms timing accuracy of $100 \mathrm{~ns}$ and if 10 year observation of 300 pulsars with $100 \mathrm{~ns}$ timing accuracy would probe graviton masses down to $3 \times 10^{-23} \mathrm{eV}\left(\lambda_{g}=4.1 \times 10^{13} \mathrm{~km}\right)$.

Aim of our investigation is that our previous results [62] about the constraints on parameters of Yukawa gravity from observations of trajectories of bright stars near the Galactic Center use to obtain a graviton mass bounds.

\section{Graviton mass estimates from S2 star orbit}

Monitoring bright stars at the Galactic Center is given in the following references [67-75]. The astrometric observations of S2 star were given in the references [76]. Recently, they were used to evaluate parameters of black hole and to test and constrain several models of modified gravity at mpc scales [77-81]. Comparisons of results obtained in Yukawa gravity model with the NTT/VLT astrometric observations of S2 star [76] resulted with the constraints on the range of Yukawa interaction $\lambda$ [62]. Comparison showed that $\lambda$ is most likely on the order of several thousand astronomical units $\lambda \sim 5000-6000 \mathrm{AU}$. This result can be used to constrain the lower bound for Compton wavelength $\lambda_{g}$ of the graviton, and thus the upper bound for its mass $m_{g}=h c / \lambda_{g}$, assuming Yukawa gravitational potential of a form $\propto r^{-1} \exp \left(-r / \lambda_{g}\right)$ [see e.g. 31]. We find these constraints using chi-square test of goodness of the $\mathrm{S} 2$ star orbit fits by Yukawa gravity potential (1).

In order to obtain the reliable statistical criterion,we used the following expression:

$$
\chi^{2}=\sum_{i=1}^{n}\left[\frac{\left(x_{i}^{o}-x_{i}^{c}\right)^{2}}{\sigma_{x i}^{2}+\sigma_{i n t}^{2}}+\frac{\left(y_{i}^{o}-y_{i}^{c}\right)^{2}}{\sigma_{y i}^{2}+\sigma_{i n t}^{2}}\right],
$$

where $\left(x_{i}^{o}, y_{i}^{o}\right)$ is the $i$-th observed position, $\left(x_{i}^{c}, y_{i}^{c}\right)$ is the corresponding calculated position, $n$ is the number of observations, $\sigma_{x i}$ and $\sigma_{y i}$ are uncertainties of observed positions, while $\sigma_{i n t}$ accounts for the "intrisic dispersion" of the data [84]. $\sigma_{\text {int }}$ is usually introduced whenever the observed uncertainties are not mutually consistent, like it is the case with SN Ia data in cosmology (see e.g. [82, 83] and references therein).

We performed the fits of $n=70$ positions of S2 star observed by NTT/VLT [76] with its simulated orbits in Yukawa gravity potential (1), by varying $\lambda$ between 1500 and $20000 \mathrm{AU}$. We assume value 


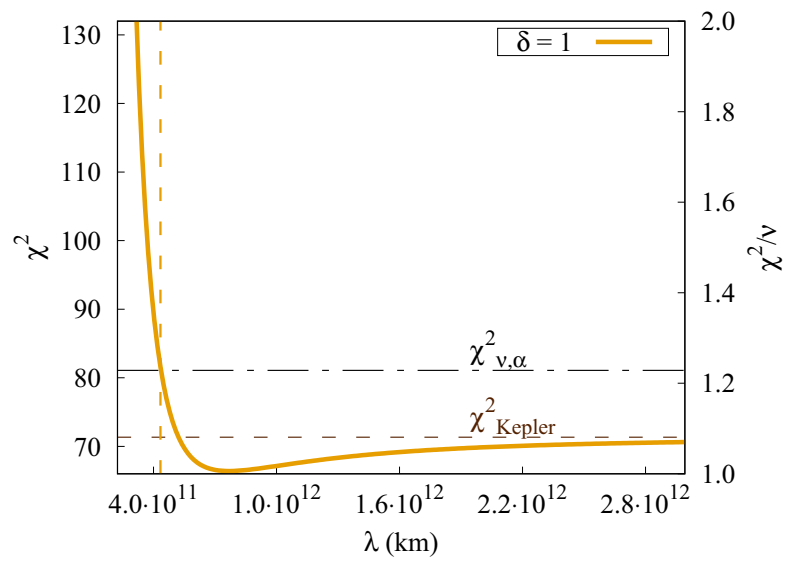

Figure 1. $\chi^{2}$ (solid lines) as a function of Yukawa range of interaction $\lambda$, i.e. the graviton Compton wavelength $\lambda_{g}$, obtained from the fits of NTT/VLT observations of S2 star [76] using the gravity potential (1) for $\delta=1$. For comparison, the value of the Keplerian fit $\chi_{\text {Kepler }}^{2}=71.34$ is also denoted by the horizontal dashed line. The critical value for $v=66$ degrees of freedom and $\alpha=0.1$ significance level, $\chi_{v, \alpha}^{2}=81.08$, is presented by the horizontal dash-dotted line, and the upper bounds $\lambda_{x}$ of the corresponding exclusion regions for $\lambda_{g}$ by the vertical dotted lines. The values $\lambda_{g}<\lambda_{x}$ can be excluded with $90 \%$ probability.

of $\delta=1$. In total 4 parameters were fitted: two components of initial position and two components of initial velocity in orbital plane. The number of degrees of freedom is $v=70-4=66$ [62]. Fitting is performed by minimization of $\chi^{2}$ (see 2), where $\sigma_{i n t}$ is estimated from the requirement that the global minimum of reduced $\chi^{2}$ over the whole range of $\lambda$ is $\chi^{2} / v=1$ (see Fig. 1). We obtained that $\sigma_{\text {int }}=1.13$ mas satisfies this requirement.

The resulting value of $\chi^{2}$ for $\delta=1$ as functions of $\lambda$ is presented in Fig. 1. We can see that $\chi^{2}$ asymptotically approaches to the corresponding value of the Keplerian fit $\chi_{\text {Kepler }}^{2}=71.34$ (horizontal dashed line in Fig. 1), for the large values of $\lambda$. Also, $\chi^{2}$ has global minimum at $\lambda=5100 \pm 50 \mathrm{AU} \approx$ $7.6 \times 10^{11} \mathrm{~km}$ in the case of $\delta=1$. We can conclude that the obtained results for $\lambda$ are consistent with those from [62].

Further, we use the obtained values of $\chi^{2}$ to test the null hypothesis that $\lambda$ should be at least on the order of $10^{3} \mathrm{AU}$. In order to test this hypothesis we assumed the significance level $\alpha=0.1$ and we calculated the critical value $\chi_{v, \alpha}^{2}=81.08$ for $v=66$ degrees of freedom (horizontal dash-dotted line in Fig. 1). As it can be seen from Fig. 1, there is an exclusion range of $\lambda$ with some upper bound $\lambda_{x}$ where $\chi^{2}>\chi_{v, \alpha}^{2}$, so the cases $\lambda<\lambda_{x}$ can be excluded with very high probability of $1-\alpha=90 \%$. This upper exclusion bound is $\lambda_{x}=2900 \pm 50 \mathrm{AU} \approx 4.3 \times 10^{11} \mathrm{~km}$. Since the null hypothesis can be considered as valid for $\lambda>\lambda_{x}$, this value can be taken as the lower allowed bound for the graviton Compton wavelength $\lambda_{g}>\lambda_{x}$. Our results are in agreement with Solar System and LIGO constraints on $\lambda_{g}$ (see Fig. 8 from [2]).

We obtain that the corresponding upper bound for graviton mass, $m_{g}=h c / \lambda_{x}$, is $m_{g}=2.9 \times$ $10^{-21} \mathrm{eV}$ in the case of $\delta=1$ [84]. These constraints are consistent with those obtained from a gravitation wave signal GW150914 [2]. It significantly exceeds $1.2 \times 10^{-22} \mathrm{eV}$ which represents $90 \%$ probability limit for distinguishing massless gravitons from massive gravitons using pulsar timing experiments [see e.g. 63]. 


\section{Conclusions}

We consider phenomenological consequences of massive gravity and show that an analysis of bright star trajectories could bound the graviton mass. We perform simulations of the S2 star orbit around the supermassive black hole at the Galactic Center in Yukawa gravity [62] and we compare our results with the NTT/VLT astrometric observations of S2 star [76]. We obtain the constraints on the range of Yukawa interaction $\lambda>4.3 \times 10^{11} \mathrm{~km}$ and corresponding most likely upper bound for graviton mass $m_{g}<2.9 \times 10^{-21} \mathrm{eV}$.

Our estimate of a graviton mass is slightly greater than the estimate obtained by the LIGO collaboration with the first detection of gravitational waves from the binary black hole system. We can conclude that a) our estimate is consistent with the LIGO estimations; b) our estimation is based on trajectories analysis of bright stars near the Galactic Center; c) in the future our estimate may be improved with forthcoming observational facilities like GRAVITY [85], E-ELT [86] and TMT [87]. We believe that in the future such facilities may improve the discussed estimates of graviton mass.

A.F.Z. thanks the organizers of Quarks-2016 for their kind and invitation to present the talk at the seminar. A.F.Z. appreciates also NSF (HRD-0833184) and NASA (NNX09AV07A) at NASA CADRE and NSF CREST Centers (NCCU, Durham, NC, USA) for a partial support. P.J., D.B.and V.B.J. wish to acknowledge the support by the Ministry of Education, Science and Technological Development of the Republic of Serbia through the project 176003 "Gravitation and the large scale structure of the Universe".

\section{References}

[1] B. P. Abbott et al., (LIGO Scientific Collaboration and Virgo Collaboration), Phys. Rev. Lett. 116, 061102 (2016).

[2] B. P. Abbott et al., (LIGO Scientific Collaboration and Virgo Collaboration), LIGO Document P1500213-v27, arXiv:1602.03841 (2016).

[3] A. Einstein, Sitzungsber. K. Preuss. Akad. Wiss. 844 (1915).

[4] D. Hilbert, Göttingen Nachr. 3, 395 (1915).

[5] J. Mehra, Einstein, Hilbert and the Theory of Gravitation, (Dordrecht, Boston, 1974).

[6] J. Earman, C. Glymour Arch. Hist. and Exact. Sci. 19, 291 (1978).

[7] V.P. Vizgin, Ya.A. Smorodinskii, Physics - Uspekhi 22, 489 (1979).

[8] V.P. Vizgin, Physics - Uspekhi 44, 1283 (2001).

[9] A.A. Logunov, M.A. Mestvirishvili, V.A. Petrov, Physics - Uspekhi 47, 607 (2004).

[10] A. Einstein, Sitzungsber. K. Preuss. Akad. Wiss. 1, 688 (1916).

[11] V. P. Vizgin, V. Ya. Frenkel, in Einstein's collection 1983- 1984 (Einteinovskij sbornik 19831984), 106 (1988) (in Russian).

[12] A. S. Sonin, V. Ya. Frenkel, Priroda 10, 86 (1994) (in Russian).

[13] A. S. Sonin, V. Ya. Frenkel, Vsevolod Konstantinovich Frederiks, (Nauka, Moscow, 1995) (in Russian).

[14] C.E. Guye, S. Ratnowsky, Comptes Rendus de l' Académie 150326 (1910).

[15] C.E. Guye, C. Lavanchy, Comptes Rendus de l' Académie 16152 (1915).

[16] C.E. Guye, C. Lavanchy, Comptes Rendus de l'Académie 42286 (1916).

[17] W. Voigt, Nachrichten von der Königl. Gesellschaft der Wissenschaften und der Georg-AugustsUniversität zu Göttingen, 41 (1887).

[18] A. Ernst, J.-P. Hsu, Chinese J. Phys. 39, 211 (2001). 
[19] A. Ernst, J.-P. Hsu, in Lorentz and Poincare invariance, ed. J.-P. Hsu (World Scientific, Singapore, 2001), p. 4.

[20] W. Voigt, V. Freedericksz, Annalen der Physik 353, 145 (1915).

[21] W. C. Röntgen, Wied. Ann. 8916 (1889).

[22] V. K. Frederiks, Uspekhi Fiz. Nauk 2, 162 (1921) (in Russian); reprinted in Phys. - Usp. 42, 1235 (1999).

[23] V. K. Frederiks, A.A. Friedmann, Tensor calculus (Leningrad, Academia, 1921) (in Russian).

[24] V. A. Fock, Sov. Phys. Usp. 6, 473 (1964).

[25] A.A. Friedmann, Zeitschrift für Physik 10, 377 (1922); English translation in General Relativity and Gravitation 31, 1991 (1999).

[26] A.A. Friedmann, Zeitschrift für Physik 21, 326 (1924); English translation in General Relativity and Gravitation 31, 2001 (1999)

[27] G. Gamow, Phys. Rev. 70, 572 (1946).

[28] E. A. Tropp, V. Ya. Frenkel, A. D. Chernin, Alexander A. Friedmann: The man who made the Universe expand, (Cambridge University Press, 2006).

[29] B. P. Abbott et al., (LIGO Scientific Collaboration and Virgo Collaboration), Phys. Rev. Lett. 116, 241103 (2016).

[30] M. Fierz and W. Pauli, Proc. of the Royal Society of London, Series A 173, 211 (1939).

[31] C. Will, Phys. Rev. D 572061 (1998); [gr-qc/9709011].

[32] C. Will, Living Reviews in Relativity 17, 4 (2014); arXiv:1403.7377v1[gr-qc].

[33] J. D. Anderson, P. A. Laing, E. L. Lau, A. S. Liu, M. M. Nieto and S. G. Turyshev, Phys. Rev. Lett. 81, 2858 (1998).

[34] J. D. Anderson, J. K. Campbell, J. E. Ekelund, J. Ellis and J. F. Jordan, Phys. Rev. Lett. 100, 091102 (2008).

[35] S. G. Turyshev, V. T. Toth, G. Kinsella, S. C. Lee, S. M. Lok and J. Ellis, Phys. Rev. Lett. 108, 241101 (2012).

[36] V. I. Zakharov, JETP Letters 12, 447 (1970).

[37] H. van Dam and M. Veltman, Nucl. Phys. B 22, 397 (1970) .

[38] Y. Iwasaki, Phys. Rev. D 2, 2255 (1970).

[39] V. A. Rubakov and P. G. Tinyakov, Physics - Uspekhi 51, 759 (2008).

[40] A. I. Vainshtein, Phys. Lett. B 39, 393 (1972).

[41] M. Visser, Gen. Rel. Grav. 30, 1717 (1998).

[42] G. Dvali, G. Gabadadze and M. Porrati, Phys. Lett. B 485, 112 (2000).

[43] I. I. Kogan, S. Mouslopoulos, A. Papazoglou and G. G. Ross, Nucl. Phys. B 595, 225 (2001).

[44] C. Deffayet, G. Dvali, G. Gabadadze and A. Vainshtein, Phys. Rev. D 65, 044026 (2002).

[45] L. S. Finn and P. J. Sutton, Phys. Rev. D 65, 044022 (2002).

[46] T. Damour, I. I. Kogan and A. Papazoglou, Phys. Rev. D 67, 064009 (2003).

[47] M. Maggiorre, Gravitational Waves, Vol. VI, (Oxford University Press, 2008).

[48] K. Hinterbichler, Rev. Mod. Phys. 84, 671 (2012).

[49] S. Capozziello, A. Stabile and A. Troisi, Mod. Phys. Lett. A 24, 659 (2009).

[50] A. S. Goldhaber and M. M. Nieto, Phys. Rev. D 9, 1119 (1974).

[51] P. G. O. Freund, A. Maneshwari and E. Schonberg, Astrophys. J. 157, 857 (1969).

[52] A. A. Logunov and M. A. Mestvirishvili, Theor. Math. Phys. 61, 1170 (1984).

[53] S. S. Gershtein, A. A. Logunov and M. A. Mestvirishvili, Physics - Uspekhi 49, 1179 (2006). 
[54] S. S. Gershtein, A. A. Logunov and M. A. Mestvirishvili, Phys. Part. Nucl. 39, 1 (2008).

[55] A. S. Goldhaber and M. M. Nieto, Rev. Mod. Phys. 82, 939 (2010).

[56] S. M. Kopeikin, Astrophys. J. Lett. 556, L1 (2001); [gr-qc/0105060].

[57] E. B. Fomalont and S. M. Kopeikin, Astrophys. J. 598, 704 (2003); [astro-ph/0302294].

[58] S. M. Kopeikin, Class. Quant. Grav. 21, 3251 (2004), [gr-qc/0310059].

[59] S. M. Kopeikin, Class. Quantum Grav. 22, 5181 (2005); [gr-qc/0501048].

[60] S. M. Kopeikin, Int. J. Mod. Phys. D 15, 273 (2006); [gr-qc/0501001].

[61] C. Talmadge, J.-P. Berthias, R. W. Hellings and E. M. Standish, Phys. Rev. Lett. 61, 1159 (1988).

[62] D. Borka, P. Jovanović, V. Borka Jovanović, A. F. Zakharov, J. Cosm. Astropart. Phys. 11, 050 (2013).

[63] K. J. Lee, F. A. Jenet, R. H. Price, N. Wex and M. Kramer, Astrophys. J. 722, 1589 (2010).

[64] M. V. Sazhin, Sov. Astron. 22, 36 (1978).

[65] F. A. Jenet, G. B. Hobbs, K. J. Lee and R. N. Manchester, Astrophys. J. 625, L123 (2005).

[66] K. J. Lee, F. A. Jenet and R. H. Price, Astrophys. J. 685, 1304 (2008).

[67] A. M. Ghez, M. Morris, E. E. Becklin, A. Tanner and T. Kremenek,Nature 407, 349 (2000).

[68] G. F. Rubilar and A. Eckart, Astron. Astrophys. 374, 95 (2001).

[69] R. Schödel, T. Ott, R. Genzel et al., Nature 419, 694 (2002).

[70] A. M. Ghez, G. Duchêne, K. Matthews et al., Astrophys. J. Lett. 586, L127 (2003).

[71] A. M. Ghez, S. A. Wright, K. Matthews et al., Astrophys. J. Lett. 601, L159 (2004).

[72] A. M. Ghez, S. Salim, N. N. Weinberg et al., Astrophys. J. 689, 1044 (2008).

[73] S. Gillessen, F. Eisenhauer, S. Trippe et al., Astrophys. J. 692, 1075 (2009).

[74] S. Gillessen, R. Genzel, T. K. Fritz et al., Nature, 481, 51 (2012).

[75] L. Meyer, A. M. Ghez, R. Schödel et al., Science, 338, 84 (2012).

[76] S. Gillessen, F. Eisenhauer, T. K. Fritz et al., Astrophys. J. 707, L114 (2009).

[77] A. F. Zakharov, A. A. Nucita, F. De Paolis and G. Ingrosso, Phys. Rev. D 76, 062001 (2007).

[78] A. F. Zakharov, S. Capozziello, F. De Paolis, G. Ingrosso and A. A. Nucita, Space Sci. Rev. 48, 301 (2009).

[79] D. Borka, P. Jovanović, V. Borka Jovanović and A. F. Zakharov, Phys. Rev. D 85, 124004 (2012).

[80] A. F. Zakharov, J. Astrophys. Astron. 36, 539 (2015).

[81] A. F. Zakharov, in Gravitation, Astrophysics, and Cosmology - Proc. Twelfth Asia-Pacific International Conference, edited by J.-P. Hsu and V.N. Melnikov (World Scientific Publ., Singapore, 2016) p. 176.

[82] P. Astier, J. Guy, N. Regnault et al., Astron. Astrophys. 447, 31 (2006).

[83] A. F. Zakharov and V. N. Pervushin, Phys. Atom. Nucl. 75, 1418 (2012).

[84] A. F. Zakharov, P. Jovanović, D. Borka, V. Borka Jovanović, J. Cosm. Astropart. Phys. 05, 045 (2016).

[85] N. Blind, F. Eisenhauer, S. Gillessen et al., arXiv:1503.07303 [astro-ph.IM].

[86] A. Ardeberg, J. Bergeron, A. Cimatti et al., An Expanded View of the Universe Science with the European Extremely Large Telescope, ed. by M. Lyubenova and M. Kissler-Patig, ESO, Munich.

[87] W. Skidmore et al., Thirty Meter Telescope Detailed Science Case: 2015 (TMT Observatory Corporation). 